\title{
Two New Closed Form Approximate Maximum Likelihood Location Methods Based on Time Difference of Arrival Measurements
}

\author{
Wanchun $\mathrm{Li}^{1,2}$, Qiu Tang ${ }^{1}$, Yingxiang $\mathrm{Li}^{2}$ and Guobin Qian ${ }^{1}$ \\ ${ }^{1}$ School of Electronic Engineering, University of Electronic Science and Technology of China, Chengdu 611731 \\ ${ }^{2}$ School of Communication Engineering, Chengdu University of Information Technology, Meteorological information and Signal \\ Processing Key Laboratory of Sichuan Higher Education Institutes Chengdu 611731
}

\begin{abstract}
In this paper, we propose two new closed form approximate maximum likelihood location methods via time difference of arrival (TDOA) measurements to determine the location of a target. For both two methods, an initial estimation is acquired by least square method in the first step. Then, supposing that the statistical structure of measurement noise are already known, the maximum likelihood function is derived. At last, for method one, we use first order Taylor expand at the initial point to approximate the residual error for nonlinear measurement equation and substitute it into the maximum likelihood function. While for method two, we use second order Taylor expand at the initial point to approximate the maximum likelihood function. At last, we derive the closed-form solution to both of the methods respectively. The computational complexity of our methods and AML method is derived and we analyze the performance of our two methods. The simulation results show that our methods are more accurate because our methods based on maximum likelihood function and have low computational complexity because we iterate only once.
\end{abstract}

Keywords-TDOA location; maximum likelihood; closed form solution; performance analysis

\section{INTRODUCTION}

The past few decades, the problem of determining the position of moving or static object(s) by measuring time difference of arrival (TDOA) of received signals has drawn considerable attention, and research on TDOA technologies has made great progress. Now, TDOA technologies are widely used in many areas, including radar, mobile communications, GPS and wireless sensor networks [1-8].

TDOA technologies have been used for target location for a long time, and many algorithms have been proposed. In [9], in order to handle nonlinear location equations, author employs Taylor series expansion so as to linearize it. Unlike [9], we apply Taylor expansion to approach the maximum likelihood equation rather than the location equations. Our methods also have less computational complexity because they need iteration procedure only once. In [10], authors use least-square solution, named as spherical-interpolation (SI). In [11], authors relax the cost function as minimum and maximum function and use two different SDR methods [11-14] for relaxation. In [15], authors propose a bi-iterative method to determine the location or speed of a moving target alternately. Authors derive a closed-form solution through the WLS expression and employ Gauss-Newton method for a better solution. While our methods derive the closed-form solution through maximum likelihood equation.

In [16], Chan proposed a classical method to manage TDOA problem. When the error of TDOA is small enough, it can attain to CRLB, but when error is large, the performance of the algorithm is not guaranteed. In [6], in order to deal with the non-linear equation, authors apply the Levenberg-Marquardt algorithm and meet great balance between accuracy and computational complexity. Since the Chan method is biased when the measurement noise is large, authors of [17] propose two methods to reduce the bias considerably and achieve the CRLB for distant source location problem.

In [18], authors first change the maximum likelihood equations into two linear equations which coefficients are about unknowns target coordinates $(x, y)$, with some initial values of $(x, y)$, the approximate maximum likelihood (AML) solves the linear equations for new $(x, y)$ and updates their coefficients. After five updates, AML selects the $(x, y)$ that minimize the cost function as the solution.

Our method also starts from a maximum likelihood function, unlike [18], we do not need to iterate, which reduces the computation load. Applying the initial guess derived from the least squares method and final results can be derived through a maximum likelihood based closed form solution.

The arrangement of this paper lies follows: section II gives the derivation of our methods, The computer simulation results are shown in section III and our conclusion is drawn in section IV. At last, we analyze the bias and variance for least square location method in section VII.

\section{Proposed Method}

In this section, the model and the derivation of our two methods are presented. In this paper, we propose our two methods to determine an individual target position. The measurements we use are the time difference of target-receiver distance. Since the parameter extraction procedure is not considered in our paper, we do not care whether the target itself transmits signal or is illuminated by other kinds of 
signals. As for receivers, we have their priori knowledge and know their position precisely.

In TDOA location, we have the measurement equations:

$$
\tau_{k 1}=\frac{1}{C}\left(R_{k}-R_{1}\right) \quad k=2,3, \cdots, M
$$

Where:

$$
R_{k}=\sqrt{\left(\boldsymbol{\theta}-\mathbf{x}_{k}\right)^{T}\left(\boldsymbol{\theta}-\mathbf{x}_{k}\right)}
$$

Here $\boldsymbol{\theta}$ is the target position to be determined and its dimension is $K$. Suppose $K=2$, our problem is based on the $2 \mathrm{D}$ plane, and when $\mathrm{K}=3$, we locate a target in $3 \mathrm{D}$ space. For TDOA hyperbolic location, $\mathrm{K}=\mathrm{M}$ may cause fake focal point, we assume that $M \geq K+1$ to ensure a unique position solution. $\quad \mathbf{x}_{k}$ here is the $k$ th receiver position and $R_{k}$ represents the distance between target and $k$ th receiver. For simplicity, we define:

$$
\rho_{k}=c \tau_{k 1} \quad k=2,3, \cdots, M
$$

Thus:

$$
\rho_{k}+R_{1}=R_{k}
$$

Squaring both sides of the equation (4) and get:

$$
\rho_{k}^{2}+2 R_{1} \rho_{k}+R_{1}^{2}=R_{k}^{2}
$$

Substitute (2) into (5) and obtain:

$$
\rho_{k}^{2}+2 R_{1} \rho_{k}+2\left(\mathbf{x}_{k}-\mathbf{x}_{1}\right)^{T} \boldsymbol{\theta}+\mathbf{x}_{1}^{T} \mathbf{x}_{1}-\mathbf{x}_{k}{ }^{T} \mathbf{x}_{k}=0
$$

Where $\boldsymbol{\theta}$ and $R_{1}$ are unknowns. Note that equation (6) is a series of equations related to subscript from 2 to $\mathrm{M}$. We can rewrite it in the form of matrix as:

$$
\mathbf{A u}=\mathbf{b}
$$

Where

$$
\mathbf{A}=2\left[\begin{array}{cc}
\mathbf{x}_{2}{ }^{T}-\mathbf{x}_{1}{ }^{T} & \rho_{2} \\
\mathbf{x}_{3}{ }^{T}-\mathbf{x}_{1}{ }^{T} & \rho_{3} \\
\vdots & \vdots \\
\mathbf{x}_{M}{ }^{T}-\mathbf{x}_{1}{ }^{T} & \rho_{M}
\end{array}\right] \mathbf{b}=\left[\begin{array}{c}
\rho_{2}{ }^{2}+\mathbf{x}_{1}{ }^{T} \mathbf{x}_{1}-\mathbf{x}_{2}{ }^{T} \mathbf{x}_{2} \\
\rho_{3}{ }^{2}+\mathbf{x}_{1}{ }^{T} \mathbf{x}_{1}-\mathbf{x}_{3}{ }^{T} \mathbf{x}_{3} \\
\vdots \\
\rho_{M}{ }^{2}+\mathbf{x}_{1}{ }^{T} \mathbf{x}_{1}-\mathbf{x}_{M}{ }^{T} \mathbf{x}_{M}
\end{array}\right] \mathbf{u}=\left[\begin{array}{c}
\boldsymbol{\theta} \\
R_{1}
\end{array}\right]
$$

Notice that $\boldsymbol{\theta}$ and $R_{1}$ are related to each other. Thus, a better solution requires decorrelation later, such as literature [16].

In receiver, measurements noise is inevitable. We model noisy TDOA measurements as:

$$
\hat{\boldsymbol{\rho}}=\boldsymbol{\rho}+\mathbf{n}
$$

Where

$$
\begin{aligned}
& \hat{\boldsymbol{\rho}}=\left[\begin{array}{lll}
\hat{\rho}_{2} & \cdots & \hat{\rho}_{M}
\end{array}\right]^{T} ; \quad \boldsymbol{\rho}=\left[\begin{array}{lll}
\rho_{2} & \cdots & \rho_{M}
\end{array}\right]^{T} \\
& \mathbf{n}=\left[\begin{array}{lll}
n_{2} & \cdots & n_{M}
\end{array}\right]^{T}
\end{aligned}
$$

Normally, measurements noise $\mathbf{n}$ is zero mean Gaussian noise and its covariance matrix is $\mathbf{Q}$. Therefore, its probability density function is written as:

$$
p(n \mid \boldsymbol{\theta})=\frac{1}{(2 \pi)^{\frac{M}{2}}|\mathbf{Q}|^{\frac{1}{2}}} \exp \left\{-\frac{1}{2}[\hat{\boldsymbol{\rho}}-\boldsymbol{\rho}(\boldsymbol{\theta})]^{T} \mathbf{Q}^{-1}[\hat{\boldsymbol{\rho}}-\boldsymbol{\rho}(\boldsymbol{\theta})]\right\}
$$

The likelihood function of (11) is represented as:

$$
\begin{aligned}
& L(n \mid \boldsymbol{\theta})=\ln p(n \mid \boldsymbol{\theta})=-\frac{M}{2} \ln (2 \pi)-\frac{1}{2} \ln |\mathbf{Q}| \\
& -\frac{1}{2}[\hat{\boldsymbol{\rho}}-\boldsymbol{\rho}(\boldsymbol{\theta})]^{T} \mathbf{Q}^{-1}[\hat{\boldsymbol{\rho}}-\boldsymbol{\rho}(\boldsymbol{\theta})]
\end{aligned}
$$

Omit the constant term and obtain the maximum likelihood solution to it as:

$$
\hat{\boldsymbol{\theta}}_{M L}=\min _{\boldsymbol{\theta}} l(\boldsymbol{\theta})
$$

Where

$$
l(\boldsymbol{\theta})=[\hat{\boldsymbol{\rho}}-\boldsymbol{\rho}(\boldsymbol{\theta})]^{T} \mathbf{Q}^{-1}[\hat{\boldsymbol{\rho}}-\boldsymbol{\rho}(\boldsymbol{\theta})]
$$

For the sake of solving (14), we make use of the Taylor series location method $[9,15]$ and iterate for the final solution. But the iteration needs a good initial value to void divergence, so we apply least square method to (7) so as to get the initial guess:

$$
\hat{\mathbf{u}}_{L S}=\left(\hat{\mathbf{A}}^{T} \hat{\mathbf{A}}\right)^{-1} \hat{\mathbf{A}}^{T} \hat{\mathbf{b}}
$$

Where the first two row of $\hat{\mathbf{u}}_{L S}$ is the original value

$$
\hat{\boldsymbol{\theta}}_{L S}=\hat{\mathbf{u}}_{L S}(1: 2)
$$

After we acquire the initial value, we propose two methods for the final determination of target location.

\section{A. Proposed Method 1}

Taylor expand $\boldsymbol{\rho}(\boldsymbol{\theta})$ at $\hat{\boldsymbol{\theta}}_{L S}$ and ignore terms more than second order and obtain:

$$
\boldsymbol{\rho}(\boldsymbol{\theta})=\boldsymbol{\rho}\left(\hat{\boldsymbol{\theta}}_{L S}\right)+\mathbf{F}\left(\boldsymbol{\theta}-\hat{\boldsymbol{\theta}}_{L S}\right)
$$


Where

$$
\mathbf{F}=\left.\frac{\partial \boldsymbol{\rho}}{\partial \boldsymbol{\theta}^{T}}\right|_{\boldsymbol{\theta}=\hat{\boldsymbol{\theta}}_{L S}}
$$

Substituting (17) into (13), yields:

$$
\hat{\boldsymbol{\theta}}_{M L}=\min _{\boldsymbol{\theta}}\left[\tilde{\boldsymbol{\rho}}-\mathbf{F}\left(\boldsymbol{\theta}-\hat{\boldsymbol{\theta}}_{L S}\right)\right]^{T} \mathbf{Q}^{-1}\left[\tilde{\boldsymbol{\rho}}-\mathbf{F}\left(\boldsymbol{\theta}-\hat{\boldsymbol{\theta}}_{L S}\right)\right]
$$

Where

$$
\tilde{\boldsymbol{\rho}}=\hat{\boldsymbol{\rho}}-\boldsymbol{\rho}\left(\hat{\boldsymbol{\theta}}_{L S}\right)
$$

Solving (19) and obtain the closed-form solution as:

$$
\hat{\boldsymbol{\theta}}_{M L}=\hat{\boldsymbol{\theta}}_{L S}+\left(\mathbf{F}^{T} \mathbf{Q}^{-1} \mathbf{F}\right)^{-1} \mathbf{F}^{T} \mathbf{Q}^{-1} \tilde{\boldsymbol{\rho}}
$$

\section{B. Proposed Method 2}

Taylor expand likelihood function (14) at $\boldsymbol{\theta}=\hat{\boldsymbol{\theta}}_{L S}$ and omit high-order terms more than third order and get:

$$
\begin{aligned}
& l(\boldsymbol{\theta})=l\left(\hat{\boldsymbol{\theta}}_{L S}\right)+\boldsymbol{\beta}^{T}\left[\hat{\boldsymbol{\rho}}-\boldsymbol{\rho}\left(\hat{\boldsymbol{\theta}}_{L S}\right)\right] \\
& +\frac{1}{2}\left[\hat{\boldsymbol{\rho}}-\boldsymbol{\rho}\left(\hat{\boldsymbol{\theta}}_{L S}\right)\right]^{T} \boldsymbol{\Omega}\left[\hat{\boldsymbol{\rho}}-\boldsymbol{\rho}\left(\hat{\boldsymbol{\theta}}_{L S}\right)\right]
\end{aligned}
$$

Where

$$
\begin{aligned}
& \boldsymbol{\beta}=\left.\frac{\partial l(\boldsymbol{\theta})}{\partial \boldsymbol{\theta}}\right|_{\boldsymbol{\theta}=\hat{\boldsymbol{\theta}}_{L S}}=\mathbf{F}^{T} \mathbf{Q}^{-1}\left[\hat{\boldsymbol{\rho}}-\boldsymbol{\rho}\left(\hat{\boldsymbol{\theta}}_{L S}\right)\right] \\
& \boldsymbol{\Omega}=\left.\frac{\partial l^{2}(\boldsymbol{\theta})}{\partial \boldsymbol{\theta} \partial \boldsymbol{\theta}^{T}}\right|_{\boldsymbol{\theta}=\hat{\boldsymbol{\theta}}_{L S}=\mathbf{J}+\mathbf{S}}
\end{aligned}
$$

Where

$$
\begin{gathered}
\mathbf{S}=\sum_{i=2}^{M} \sum_{j=2}^{M}\left(\phi_{i j} r_{j} \frac{\partial^{2} r_{i}}{\partial \boldsymbol{\theta} \partial \boldsymbol{\theta}^{T}}+r_{i} \phi_{i j} \frac{\partial^{2} r_{j}}{\partial \boldsymbol{\theta} \partial \boldsymbol{\theta}^{T}}\right) \\
\mathbf{J}=\sum_{i=2}^{M} \sum_{j=2}^{M}\left(\phi_{i j} \frac{\partial r_{j}}{\partial \boldsymbol{\theta}} \frac{\partial r_{i}}{\partial \boldsymbol{\theta}^{T}}+r_{i} \phi_{i j} \frac{\partial r_{i}}{\partial \boldsymbol{\theta}} \frac{\partial r_{j}}{\partial \boldsymbol{\theta}^{T}}\right)=2 \mathbf{F}^{T} \mathbf{Q}^{-1} \mathbf{F} \\
\frac{\partial r_{i}}{\partial \boldsymbol{\theta}}=\left[\begin{array}{l}
\left.\frac{x-x_{1}}{\sqrt{\left(x-x_{1}\right)^{2}+\left(y-y_{1}\right)^{2}}}-\frac{x-x_{i}}{\sqrt{\left(x-x_{1}\right)^{2}+\left(y-y_{1}\right)^{2}}}-\frac{y-y_{i}}{\sqrt{\left(x-x_{i}\right)^{2}+\left(y-y_{i}\right)^{2}}}\right]_{\substack{x=\hat{i}_{L S} \\
y=y_{L S}}} \\
\phi_{i j}=\left(\mathbf{Q}^{-1}\right)_{i j}
\end{array}\right.
\end{gathered}
$$

$$
\frac{\partial^{2} r_{i}}{\partial \boldsymbol{\theta} \partial \boldsymbol{\theta}^{T}}=\left[\begin{array}{ll}
r_{i \theta 11} & r_{i \theta 12} \\
r_{i \theta 21} & r_{i \theta 22}
\end{array}\right]_{x=\hat{x}_{L S}, y=\hat{y}_{L S}}
$$

And:

$$
\begin{aligned}
& r_{i \theta 11}=\frac{\left(y-y_{1}\right)^{2}}{\left[\left(x-x_{1}\right)^{2}+\left(y-y_{1}\right)^{2}\right]^{\frac{3}{2}}}-\frac{\left(y-y_{i}\right)^{2}}{\left[\left(x-x_{i}\right)^{2}+\left(y-y_{i}\right)^{2}\right]^{\frac{3}{2}}} \\
& r_{i \theta 12}=r_{i \theta 21}=\frac{-\left(x-x_{1}\right)\left(y-y_{1}\right)}{\left[\left(x-x_{1}\right)^{2}+\left(y-y_{1}\right)^{2}\right]^{\frac{3}{2}}}+\frac{\left(x-x_{i}\right)\left(y-y_{i}\right)}{\left[\left(x-x_{i}\right)^{2}+\left(y-y_{i}\right)^{2}\right]^{\frac{3}{2}}} \\
& r_{i \theta 22}=\frac{\left(x-x_{1}\right)^{2}}{\left[\left(x-x_{1}\right)^{2}+\left(y-y_{1}\right)^{2}\right]^{\frac{3}{2}}}-\frac{\left(x-x_{i}\right)^{2}}{\left[\left(x-x_{i}\right)^{2}+\left(y-y_{i}\right)^{2}\right]^{\frac{3}{2}}}
\end{aligned}
$$

The newton iteration solution thus can be represented as:

$$
\boldsymbol{\theta}_{M L_{-} \text {Newton }}=\hat{\boldsymbol{\theta}}_{L S}-\boldsymbol{\Omega}^{-1} \boldsymbol{\beta}
$$

\section{Steps for Proposed Method}

The main steps of our two methods are presented in the Table 1 and Table 2 below.

TABLE I. THE CALCULATION PROCEDURE FOR PROPOSED LOCATION METHOD 1

\begin{tabular}{|l|l|}
\hline Step 1 & $\begin{array}{l}\text { Acquire initial guess using (15), where } \mathbf{A} \text { and } \mathbf{b} \text { are } \\
\text { calculated by (8) }\end{array}$ \\
\hline Step 2 & obtain the maximum likelihood using (14) \\
\hline Step 3 & $\begin{array}{l}\text { Taylor expand } \boldsymbol{\rho}(\boldsymbol{\theta}) \text { at } \hat{\boldsymbol{\theta}}_{L S} \text { and ignore terms more than } \\
\text { second order and obtain (17) }\end{array}$ \\
\hline Step 4 & $\begin{array}{l}\text { Using the closed-form expression in (21) to get the final } \\
\text { location results }\end{array}$ \\
\hline
\end{tabular}

TABLE II. THE CALCULATION PROCEDURE FOR PROPOSED LOCATION METHOD 2

\begin{tabular}{|l|l|}
\hline Step 1 & $\begin{array}{l}\text { Acquire initial guess using (15), where } \mathbf{A} \text { and } \mathbf{b} \text { are } \\
\text { calculated by (8) }\end{array}$ \\
\hline Step 2 & obtain the maximum likelihood using (14) \\
\hline Step 3 & $\begin{array}{l}\text { Taylor expand likelihood function (14) at } \boldsymbol{\theta}=\hat{\boldsymbol{\theta}}_{L S} \text { and omit } \\
\text { high-order terms more than third order and get (22) }\end{array}$ \\
\hline Step 4 & $\begin{array}{l}\text { Using the closed-form expression in (29) to get the final } \\
\text { location results }\end{array}$ \\
\hline
\end{tabular}

\section{Simulation Results}

Monte Carlo simulations had been performed in this section to testify the performance of AML and our method comparing with CRLB.

\section{A. Performance of Different TDOA Measurement Noise} Variance

In this part, eight sensors are located at $x r(k)=R \times[\cos (k \pi / 4), \sin (k \pi / 4)], k=1,2, \ldots, 8 . \quad$ where $R$ is the radius of the circle and $R=3000 \mathrm{~m}$. The emitter is fixed at $(1000,500) m$. The TDOA measurement noises are zero-mean Gaussian white noise, and the variance of the noise 
varies from $10^{\wedge}-10 \mathrm{~m}$ to $10^{\wedge} 3.2 \mathrm{~m}$.

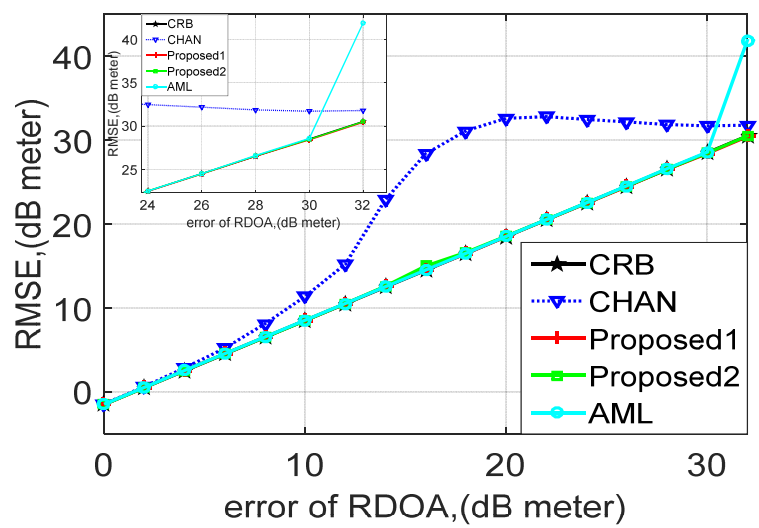

FIGURE I. THE POSITION ERROR OF DIFFERENT TDOA NOISE VARIANCE

Figure I compare the position error of different TDOA noise variance, where $\mathrm{RDOA}=10 \times \log _{10}(\mathrm{c} \times \mathrm{TDOA})$. The RMSE used in Figure $I$ is defined as $10 \times \log _{10} E\left\{\left[(\hat{x}-x)^{2}+(\hat{y}-y)^{2}\right]\right\}$. We run Monte-Carlo simulation 10000 times to draw Figure I . As we can see from the figure, CHAN method is able to achieve CRLB at moderate SNR, and it starts to derivate CRLB at around $10 \mathrm{~dB}$. Our methods, however, start to derivate at around $30 \mathrm{~dB}$ and are little better than AML method.

\section{B. Performance of Different Algorithm for Different Number of Sensors}

In this section, receivers are located at

$$
x_{m}=R \times[\cos (m \pi / 4), \sin (m \pi / 4)], m=1,2, \ldots, M .
$$

TDOA variance are fixed and set $\mathrm{M}$ from 4 to 10 to obtain table 3 .

TABLE III. THE RMSE OF DIFFERENT RECEIVERS WHEN $\delta_{\text {TDOA }}^{2}=5$

\begin{tabular}{|l|l|l|l|l|l|l|l|}
\hline$\delta_{\text {IDOA }}^{2}=5$ & 4 & 5 & 6 & 7 & 8 & 9 & 10 \\
\hline Proposed & 5.16 & 4.59 & 4.11 & 3.84 & 3.58 & 3.39 & 3.23 \\
1 & 73 & 04 & 15 & 26 & 30 & 88 & 78 \\
\hline Proposed & 5.18 & 4.58 & 4.11 & 3.84 & 3.58 & 3.39 & 3.23 \\
2 & 21 & 95 & 15 & 28 & 18 & 79 & 80 \\
\hline AML & 5.28 & 4.67 & 4.18 & 3.89 & 3.63 & 3.45 & 3.29 \\
& 61 & 15 & 33 & 80 & 21 & 47 & 50 \\
\hline CRLB & 5.15 & 4.57 & 4.16 & 3.85 & 3.60 & 3.39 & 3.22 \\
& 21 & 20 & 55 & 37 & 39 & 75 & 30 \\
\hline
\end{tabular}

TABLE IV. THE RMSE OF DIFFERENT RECEIVERS WHEN $\delta_{\text {IDDA }}^{2}=10$

\begin{tabular}{|l|l|l|l|l|l|l|l|}
\hline$\delta_{\text {TDOA }}^{2}=10$ & 4 & 5 & 6 & 7 & 8 & 9 & 10 \\
\hline Proposed1 & 10.2 & 9.222 & 8.361 & 7.773 & 7.207 & 6.815 & 6.487 \\
& 782 & 7 & 1 & 2 & 5 & 0 & 2 \\
\hline Proposed2 & 10.6 & 9.230 & 8.336 & 7.766 & 7.206 & 6.809 & 6.482 \\
& 849 & 5 & 3 & 0 & 7 & 2 & 4 \\
\hline AML & 10.4 & 9.388 & 8.464 & 7.910 & 7.291 & 6.930 & 6.607 \\
& 198 & 7 & 8 & 7 & 0 & 6 & 1 \\
\hline CRLB & 10.3 & 9.144 & 8.330 & 7.707 & 7.207 & 6.794 & 6.446 \\
& 034 & 1 & 9 & 5 & 8 & 9 & 1 \\
\hline
\end{tabular}

Table 3 and table 4 show the RMSE versus the number of sensors for different TDOA noise levels when the noise variances were identical. As can be seem from tables, our proposed method slightly outperformed AML method, and is also able to attain to CRLB.

\section{Performance of Different Algorithm for Near Field Situation (Target-receiver Distance Being Not Too Far).}

The coordinates of the ten measuring sensors are $[-42,-12],[-26,30],[-8,40],[16,18],[36,6]$, $[24,-36],[-12,24],[-20,0],[15,-15],[-30,-40] m$ and the emitter is fixe at $[0,0] \mathrm{m}$. And the noise variance ranges from $-10 \mathrm{~dB}$ to $14 \mathrm{~dB}$ so as to draw fig.

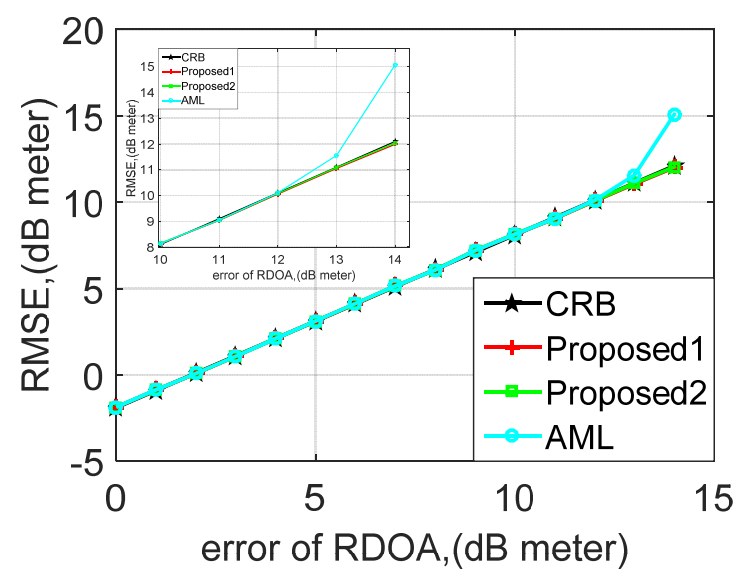

FIGURE II. RMSE OF PROPOSED TWO METHODS AND AML METHOD IN NEAR FIELD CONTEXT

Figure II compares the performance of our two methods and AML method under the near filed context. Our methods are slightly better than AML method, and proposed 1 functions even better than proposed method 2 .

\section{CONCLUSION}

In this paper, we proposed two closed-form approximate likelihood location method meant for TDOA location systems. Derivation and simulation both verify that our methods performance better than CHAN and AML method. Moreover, our methods apply Gauss-Newton iterate method only once for a better solution, which reduce the computation complexity and have less calculation load than AML method.

\section{ACKNOWLEDGMENT}

Manuscript December 13, 2017, this work was supported the Funds for the Meteorological information and Signal Processing Key Laboratory of Sichuan Higher Education Institutes, PRC.

Li WanChun, Tang Qiu and Qian GuoBin are with School of Electronic Engineering, University of Electronic Science and Technology of China, Chengdu 611731, PRC..

Li YingXiang is with School of Communication Engineering, Chengdu University of Information Technology, Meteorological information and Signal Processing Key 
Laboratory of Sichuan Higher Education Institutes Chengdu, PRC.

This work was supported by Meteorological information and Signal Processing Key Laboratory of Sichuan Higher Education Institutes (No. QXXCSYS201702).

\section{REFERENCES}

[1] Misra, P., and Enge, P.: 'Global Positioning System: Signal, measurements and performance’ (Ganga-Jamuna Press, Lincoln, MA, 2006, 2nd edn)

[2] Caffery, J.J.: 'Wireless location in CDMA cellular radio systems' (Springer, Massachusetts, USA, 2000)

[3] Patwari, N., et al.: 'Locating the nodes: cooperative localisation in wireless sensor networks’, IEEE Signal Process. Mag., 2005, 22, (4), pp. 54-69

[4] Zekavat, R., and Buehrer, R.M.: 'Handbook of position location: Theory, practice and advances' (Wiley, Natick, MA, USA, 2012, 27 ${ }^{\text {th }}$ edn)

[5] Huang, Y., Benesty, J., Elko, G.W., and Mersereau, R.M.: 'Real-time passive source localization a practical linear-correction least-squares approach', IEEE Trans. Speech Audio Process., 2001, 9, (8), pp. 943-956

[6] Mensing, C., and Plass, S.: 'Positioning algorithms for cellular networks using TDOA'. Proc. IEEE Int. Conf. on Acoustics, Speech and Signal Processing, Toulouse, France, May 2006, Vol. 4, pp. 513-516

[7] Coraluppi S.: 'Multistatic sonar localization', IEEE J. Ocean. Eng., 2006, 31, (4), pp. 964-974

[8] P. C. Chestnut, "Emitter location accuracy using TDOA and differential doppler,” IEEE Trans. Aerosp. Electron. Syst., vol. AES-18, no. 2, pp. 214-218, Mar. 1982.

[9] Wade H Foy. Position-Location Solutions by Taylor-Series Estimation [J]. IEEE Transactions on Aerospace and Electronic Systems. VOL. AES-12, NO.2 MARCH 1976.

[10] J. O. Smith and J. S. Abel, “Closed-form least-squares source location estimation from range-difference measurements," IEEE Trans. Acoust., Speech, Signal Process., vol. ASSP-35, pp. 1661-1669, Dec. 1987.

[11] ENYANG X, ZHI D, DASGUPTA S. Reduced Complexity Semidefinite Relaxation Algorithms for Source Localization Based on Time Difference of Arrival [J]. Mobile Computing, IEEE Transactions on, 2011, 10(9): 1276-82.

[12] LUI K, CHAN F, SO H C. Semidefinite Programming Approach for Range-Difference Based Source Localization [J]. Signal Processing, IEEE Transactions on, 2009, 57(4): 1630-3.

[13] LUO Z, MA W, SO A M C, et al. Semidefinite relaxation of quadratic optimization problems [J]. Signal Processing Magazine, IEEE, 2010, 27(3): 20-34.

[14] BISWAS P, LIAN T C, WANG T C, et al. Semidefinite programming based algorithms for sensor network localization [J]. ACM Transactions on Sensor Networks (TOSN), 2006, 2(2): 188-220.

[15] Guo Hui Zhu, Da Zheng Feng. Bi-iterative method for moving source localization using TDOA and FDOA measurements [J]. ELECTRONICS LETTERS, January 2015 Vol.51 No.1 pp. 8-10

[16] Chan, Y.T., and Ho, K.C.: 'A simple and efficient estimator for hyperbolic location’, IEEE Trans. Signal Process., 1994, 42, (8), pp. 1905-1915.

[17] Ho, K.C.: 'Bias reduction for an explicit solution of source localization using TDOA', IEEE Trans. Signal Process., 2012, 60, (5), pp. 2101-2114

[18] Chan, Y.T., and Huang, H.Y.C.: 'Exact and approximate maximum likelihood localisation algorithms’, IEEE Trans. Veh. Technol., 2006, 55, pp. 10-16. 